\title{
DAN HAYES
}

\section{Personal digital assistants (PDAs) for psychiatrists}

The use of personal digital assistants (PDAs), also known as palmtop or handheld computers, is becoming increasingly common in all branches of medicine. They are essentially small, mobile computers that, at the most basic level, enable you to manage your appointments, contact lists and other pertinent information. However, to dismiss them as a 'gimmicky' electronic version of that 1980s icon, the Filofax, is to miss out on the opportunity to employ a powerful and adaptable tool with capabilities that, only a few years ago, would have required constant and immediate access to a desktop computer and a small library. PDAs have the capability to manipulate information rather than passively storing it and they can run sophisticated programs (Wilcox \& La Tella, 2001). They can also download, store and update information from the internet that can then be tailored to your own personal and clinical interests.

\section{The basics}

In the continuously developing and often confusing marketplace for PDAs, a variety of factors determine the benefits that can be gained from using one.

Although the capabilities and memory, and consequently price, of PDAs tend to increase with increasing body size, a light, portable PDA that will fit unobtrusively into a pocket will certainly be able to meet the needs of all but the most demanding doctors. One of the things that has been sacrificed to ensure that some PDAs can be pocket-sized is the keyboard. Data are accessed by using a stylus to tap an appropriate icon on the screen and entered by writing in a stylised 'graffiti' font on a specified area of the screen. Although this can initially be a rather laborious process, it soon becomes relatively fast and familiar. However, if you regularly needed to write lengthy entries, particularly under a time constraint, it might be easier to use the keyboard of the 'mini-laptop' style of a larger PDA.

The amount of memory a PDA has governs how much information it can store. Most PDAs have between 8 and 16 megabytes (MB) of memory, which is likely to be adequate for most medical users. Some PDAs at the lower end of the market have $2 \mathrm{MB}$ of memory, which is likely to become a source of frustration. At the upper end of the market, machines have 32 or $64 \mathrm{MB}$ of memory.
Some PDAs also enable you to store information on removable data cards.

The operating system is the software that enables the PDA to function. Specific operating systems are linked to specific brands and there is no capacity to change. The two major operating systems are Palm OS and Microsoft Pocket PC. Devices using Palm OS tend to be smaller, lighter and cheaper and it is a system that is intuitively accessible and usable. Currently, it is also the more widely-used operating system, enabling access to a broader range of information and applications. The Pocket PC system is used on more powerful and expensive machines, and offers a greater range of functions.

Without access to a personal computer (PC), a PDA is solely reliant on the information that the owner inputs manually and is the only repository of that information. However, if it is 'synchronised' or 'hotsynced' to a PC, and both the software and 'cradle' required are supplied with PDAs, then information can be downloaded from the internet and the data you have entered onto your PDA can be 'backed up' onto your PC. Providing they share the same operating system, PDAs can also communicate with each other via an infrared link. Notes and programs can therefore be transferred readily to colleagues.

The burgeoning PDA market offers a wealth of different features. Different models come with recharging capability rather than battery power, monochrome or colour displays, keyboards, 'expansion slots' that allow you to plug in cameras, satellite navigation receivers or music MP3 players, and some allow direct internet access. Such additional capabilities obviously have cost implications, and the extent to which any or all of these features become indispensable rather than an expensive luxury depends on the individual user.

\section{PDAs for psychiatrists}

In addition to the potential benefits that PDAs offer to any busy person, they can be adapted to be relevant to psychiatrists. Doing so allows direct and convenient access to information about our own speciality that is useful on a daily basis. PDAs are equally useful for storing knowledge from other disciplines, such as medicine or surgery, which, although required less frequently, is perhaps even more welcome in an emergency situation. 
可

editorial
A visit to any internet search engine using terms like 'PDA' and 'psychiatry' will reveal a multitude of applications, predominantly of American origin. 'Portal sites' worth visiting, which give links to a wide variety of medical PDA websites, include Healthy Palmpilot (http:// www. healthypalmpilot.com), Peripheral Brain (http:// pbrain.hypermart.net), Medical Piloteer (http:// www.medicalpiloteer.com), pda MD (http:// www.pdamd.com) and the University of Alberta Library medical PDA page (http://www.library.ualberta.ca/ subject/psychiatry/pdahealth/index.cfm), although this is by no means an exhaustive list. The 'Handheld' link on the British Medical Journal (http://bmj.com/handhelds) website offers a column where colleagues can post useful website addresses.

Information or programs can be downloaded free of charge ('freeware'), for a nominal charge ('shareware') or from commercial organisations upon payment of a fee, usually by credit card payment over the internet.

Textbooks are typically only available on a commercial basis and the expanding virtual library that is available includes well-known medical textbooks and more specialist texts. Currently, there is only a small range of psychiatric textbooks available that includes such titles as the American Psychiatric Association Practice Guideline and DSM-IV text revision (http://www.handheldmed. com).

A variety of drug databases are available, with ePocrates (http://www.epocrates.com) standing out in particular. This is a free and comprehensive database that updates itself every time you synchronise your PDA. It contains a wealth of drug information including dosages, side-effects and interactions.

Specialised medical calculators such as Medmath (http://smi-web.stanford.edu/people/pcheng/ medmath/index.html) or MedCalc (http://mecalc.media.net/) are useful applications, although many of the calculations they offer will be more relevant to a physician than a psychiatrist. Anyone working with young people, especially in the field of eating disorders, will find a growth chart calculator (http://www.statcoder.com) invaluable.

PDAs can also help you keep up to date with current developments in research and practice. After registering and specifying your interests, a number of sites allow you to download articles formatted especially for your PDA. The majority of the services provided by AvantGo (http:// avantgo.com) are not medical, and the sporting or news content available may be a welcome distraction, but selecting appropriate 'channels' enables you to access subject matter from scientific or medical journals. Journal To Go (http://www.journaltogo.com) and Unbound Medicine by CogniQ (sponsored by and available through http://bmj.com) offer similar services, but are dedicated to medical journals. They both allow the selection of a variety of medical speciality channels, including 'mental health'. Content from the British Journal of Psychiatry, Psychiatric Bulletin and Advances in Psychiatric Treatment are also available in a similar format.

Psychiatric classificatory systems can be downloaded onto PDAs (DSM-IV is available from http://www. medicalpiloteer.com or http://www.handheldmed.com) and, again showing a strong American bias, 'patient tracking systems' are available that allow the clinician to keep track of patient contact, both for clinical and financial purposes.

The websites, applications and programs listed obviously do not represent a definitive view of what is available, because any attempt to be comprehensive would be both cumbersome and immediately obsolete. Psychiatrists, or indeed any other PDA users seeking to customise their machine for use in a specialist capacity, are advised to explore the range of sources available on the internet and to do so regularly. They are also encouraged to explore the flexibility of their PDA. Programs can easily be downloaded, tried out and deleted if they are unhelpful or superseded by a superior alternative. Invaluable, or even merely useful, applications can be kept and used when needed.

\section{The future}

The capabilities of PDAs already make them a valuable tool and, as their capabilities increase, it is likely that the benefits they offer will accrue. Beyond such benefits to the individual user, if their use becomes more widespread, there will also be a collective benefit to professional groups. Regular 'hotsyncing' to a hospital website could replace internal envelopes and notice boards as a way of distributing and collecting information. Educational material could be made available electronically and therefore always be accessible, even in clinical situations. The benefits of enhanced communication and information access may also be of direct clinical benefit, for example in reducing medication errors (Grasso et al, 2002).

\section{The downside}

Despite advocating the use of PDAs, it must be acknowledged that there are potential problems associated with their use. Falling prices and rising specifications mean that even a recent purchase can soon feel relatively obsolete. It must also be acknowledged that, although PDAs are generally very reliable, technology can be fallible and both hardware and software problems may occur.

As PDAs get smaller, 'losability', a familiar attribute of the mobile phone, becomes a potential concern. Beyond the inconvenience and cost of losing a PDA, there are obviously implications relating to the information stored on the machine. Obviously, the password facility that is available on PDAs to safeguard the contents should always be used, but it is also important to consider the nature of the machine's content. Tempting as it is to record patient details on a PDA, and as potentially useful as this could be, the British Medical Association warns doctors against making and keeping personal medical records on such machines, therefore this practice is to be eschewed. As well as the risk of such personal records being lost or stolen, it is important to realise they would be subject to the Data Protection Act 1998 and therefore 
would need to be made accessible to patients (http:// www.bma.org.uk/ap.nsf/content/accesshealthrecords).

This issue, particularly in the light of the availability of 'patient tracking systems' that facilitate the taking of abbreviated clinical notes on patients, highlights another potential problem with PDA use; the strong American bias of the information and applications available. More applications are becoming available from British sources (http://www.bmjbookshop.com) and further developments are promised, such as the availability of the British National Formulary in a PDA format (McFarlane \&

Connor, 2002). However, differences in medical culture and practice do exist and it should not be assumed that information derived from an American source is necessarily applicable to populations in other countries.

\section{Conclusion}

Many clinicians already use PDAs and are aware of the significant personal benefits that they offer in respect of individual time and knowledge management. More widespread use of PDAs would not only allow a greater number of individual users to appreciate these positive aspects, but would also increase the network through which knowledge could be distributed and encourage the development of useful applications.

\section{Declaration of interest}

None.

\section{References}

GRASSO, B. C., GENEST, R., YUNG, K., et devices is taking time (letter). BMJ, 325, al (2002) Reducing errors in discharge medication lists by using personal

WILCOX, R. A. \& LATELLA, R. R. (2001)

\section{3, 1325-1326.} MCFARLANE, C. \& CONNOR, E. (2002) clinical information at the point of care. medicalinstrument for the exchange of Development of BNF for handheld Medical Journal of Australia, 175, $659-662$.

Dan Hayes Specialist Registrar, Child and Adolescent Psychiatry, Children's Department, Maudsley Hospital, London SE5 8AZ 